\title{
Programmed cell death ligand 1 expression in aggressive pediatric non-Hodgkin lymphomas: frequency, genetic mechanisms, and clinical significance
}

Kevin E. Fisher,, Lizmery S. Ferguson, ${ }^{2}$ Amy M. Coffey, ${ }^{1,2,3}$ Brian Y. Merritt, Jonathan L. Curry, ${ }^{4}$ Andrea N. Marcogliese, ${ }^{1,2}$ Angela M. Major, Kala Y. Kamdar, ${ }^{5}$ Dolores H. Lopez-Terrada ${ }^{1,2,5}$ and Choladda V. Curry ${ }^{1,2}$

\begin{abstract}
'Department of Pathology and Immunology, Baylor College of Medicine, Houston; ${ }^{2}$ Department of Pathology, Texas Children's Hospital, Houston; ${ }^{3}$ Clinical Pathology Associates, Austin; ${ }^{4}$ Department of Pathology and Translational Molecular Pathology, Division of Pathology and Laboratory Medicine, The University of Texas M.D. Anderson Cancer Center, Houston and ${ }^{5}$ Department of Pediatrics, Baylor College of Medicine, Texas Children's Cancer and Hematology Centers, Houston, TX, USA
\end{abstract}

Correspondence:

Kevin E. Fisher kevin.fisher@bcm.edu Choladda V. Curry ccurry@bcm.edu

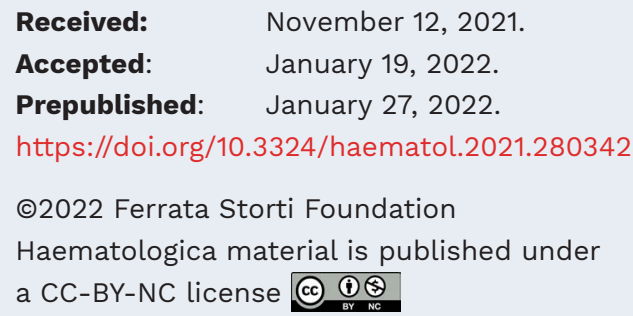

\begin{abstract}
Programmed cell death 1 (PD-1) and programmed cell death ligand 1 (PD-L1) are immunomodulatory molecules overexpressed in lymphomas and are promising immunotherapy targets for hematologic malignancies. However, studies of PD-1/PD-L1 overexpression and their clinical significance in aggressive pediatric non-Hodgkin lymphomas (NHL) are limited. We assessed PD-1/PD-L1 overexpression using immunohistochemistry in 68 aggressive pediatric NHL: ALK-positive anaplastic large cell lymphoma (ALK+ ALCL, $n=8$ ), Burkitt lymphoma ( $B L, n=27)$, and large $B$-cell lymphoma $(L B C L)$ de novo LBCL, $n=22$ and diffuse $L B C L$ arising as monomorphic post-transplant lymphoproliferative disorder [PTLD-DLBCL], $n=11$. In LBCL, correlations between PD-L1 overexpression and Epstein-Barr virus (EBV) status, cell of origin, stage, nodal status, overall survival (OS), and event-free survival (EFS) were examined. The genetic mechanisms of PD-L1 overexpression were investigated using targeted next-generation sequencing (NGS) and cytogenetic data. All ALK+ ALCL samples, 50.0\% of de novo LBCL (11/22), 72.7\% of PTLD-DLBCL (8/11), and no BL overexpressed PD-L1. Overexpressed PD-L1 correlated with EBV positivity $(P=0.033)$ in $L B C L$ and lower EFS in de novo LBCL $(P=0.017)$. NGS of select $L B C L$ revealed distinct somatic mutations and an ultra-hypermutated PTLD-DLBCL. Most cases with 9p24.1 copy gains overexpressed PD-L1 although some cases had no discernible genetic drivers of PD-L1 overexpression. Overexpressed PD-L1 is common in pediatric LBCL, associated with EBV positivity and 9p24.1 gains, and may have prognostic significance in de novo LBCL. Furthermore, diverse molecular mechanisms for PD-L1 overexpression in aggressive pediatric NHL can occur. Thus, additional studies exploring the therapeutic and prognostic significance and molecular mechanisms of PD-L1 overexpression in aggressive pediatric $\mathrm{NHL}$ are warranted.
\end{abstract}

\section{Introduction}

Immune checkpoint blockade has introduced a paradigm shift in cancer therapy for a variety of solid tumors and hematologic malignancies. ${ }^{1-3}$ Programmed cell death 1 (PD1 ) is a cell surface receptor in the B7/CD28 family of T-cell regulators that serves as an inhibitory co-receptor through binding of its ligands programmed cell death ligand 1 (PD-L1) and programmed cell death ligand 2 (PDL2). PD-L1 is broadly expressed across hematopoietic cells and human tissues, PD-1 is expressed on the cell surface of B cells, T cells, and natural killer T cells, whereas PD$L 2$ is only expressed on dendritic cells following cytokine induction. ${ }^{4,5}$ The PD-1/PD-L1/PD-L2 signaling axis promotes recognition of self and non-self antigens to maintain immune tolerance. ${ }^{5-7}$ However, tumor cells and tumor microenvironment cells can aberrantly overexpress PD-L1 (e.g., macrophages) and PD-1 (e.g., lymphocytes) to induce "T-cell exhaustion" and evade immune recognition and destruction. ${ }^{8-10}$

In adult hematologic malignancies, PD-L1 overexpression has been reported in classical Hodgkin lymphoma (cHL), 
primary mediastinal large B-cell lymphoma (PMBCL), Tcell/histiocyte-rich large B-cell lymphoma (THRLBCL), ALK-positive anaplastic large cell lymphoma (ALK+ ALCL), subsets of diffuse large B-cell lymphoma (DLBCL), and

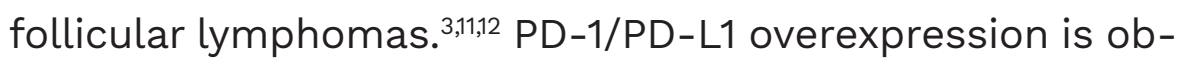
served in approximately two thirds of adults with posttransplant lymphoproliferative disorder (PTLD) following solid organ transplantation as well as a subset of EpsteinBarr virus (EBV)-driven lymphomas (e.g., EBV-positive $\mathrm{cHL}$ ). PD-1/PD-L1 expression in Burkitt lymphoma (BL) is typically negative..$^{13}$ Notably, a vast majority of these data were established in adult studies and may not be representative of pediatric populations.

Diverse molecular mechanisms drive PD-1/PD-L1 protein overexpression. For example, some tumors harbor high tumor mutation burden (TMB). This introduces numerous coding mutations that give rise to specific neoantigens that are presented to the cell surface and drive immune recognition and PD-1/PD-L1 overexpression. ${ }^{14,15}$ In adult LBCL, PD-L1 overexpression results from gene rearrangements involving the 9p24.1 gene locus which contains the CD274 (PD-L1), PDCD1LG2 (PD-L2), and JAK2 genes ${ }^{16}$ or 9p24.1 copy gains and amplifications similar to $\mathrm{CHL} .1718$ Structural variations of the CD274 gene 3' untranslated region (3'-UTR) leading to increased transcript stabilization are also reported..$^{19}$ In EBV-associated aggressive lymphomas, PD-L1 overexpression typically occurs via latent membrane protein-1 (LMP-1)-mediated activation of the CD274 JAK/STAT-dependent promoter or AP-1 associated enhancer activity. ${ }^{20,21}$ In ALK+ ALCL, PD-L1 overexpression is a result of STAT3 phosphorylation and IRF4/BATF3-mediated CD274 transcriptional upregulation. ${ }^{12,22}$

The anti-PD-1 immune checkpoint inhibitor pembrolizumab is a Food and Drug Admininistration (FDA)-approved therapeutic agent for relapsed/refractory adult and pediatric CHL. Another anti-PD-1 inhibitor, nivolumab, has shown promising therapeutic efficacy in early clinical trials for pediatric $\mathrm{CHL}$ and other PD-L1-expressing tumors. ${ }^{23,24}$ A factor contributing to the therapeutic efficacy of immune checkpoint inhibitors is the amount of target protein on the tumor tissue and/or microenvironment cells. Yet, recent evidence suggests that additional elements may also influence the use of PD-L1 expression as a predictive and prognostic marker for immune checkpoint inhibitor therapy. These may include the immunohistochemistry (IHC) methods employed, the time point at which the assessment of this dynamic biomarker is performed, TMB status, ${ }^{25,26}$ and other intrinsic biologic attributes such as DUSP22 rearrangements in ALK-negative ALCL, ${ }^{27}$ or the presence or absence of tumor infiltrating lymphocytes $(\mathrm{TIL}) .28$

Studies and clinical trials assessing PD-1/PD-L1 expression have focused primarily on adult lymphomas and pediatric $\mathrm{CHL}$. The prognostic significance of PD-L1 expression in adult DLBCL cohorts have shown conflicting results. ${ }^{29-31}$ Studies investigating PD-1/PD-L1 expression in aggressive pediatric non-Hodgkin lymphomas (NHL) such as ALCL, $\mathrm{BL}$, and de novo pediatric DLBCL are limited. Thus, we investigated PD-1/PD-L1 expression in aggressive pediatric $\mathrm{NHL}$ including $A L K+A L C L, B L$, a pediatric cohort $L B C L$ (consisting of de novo LBCL and DLBCL arising as monomorphic PTLD [PTLD-DLBCL]). In the pediatric LBCL COhort, we reviewed available cytogenetic data to assess for rearrangements involving the 9p24.1 locus and performed targeted next-generation sequencing (NGS) on selected cases to detect DNA mutations, estimate TMB, and investigate copy-number changes of the 9p24.1 locus. The impact of PD-L1 status on clinicopathologic factors and treatment outcomes in the pediatric LBCL cohort was also investigated.

\section{Methods}

\section{Case selection and clinical data}

All authors conducted the study per Institutional-approved research protocols to Drs. Curry and Fisher. Sixtyeight aggressive pediatric NHL with sufficient tissue for $I H C$ including ALK+ ALCL $(n=8), B L(n=27)$, and LBCL (consisting of de novo LBCL, $n=22$; PTLD-DLBCL, $n=11$ ) were identified via a pathology database search from 1997-2012. The term de novo LBCL was used to describe pediatric LBCL cases in our cohort arising without previous transplants or previous low-grade B-cell lymphoma. Diagnoses and further subclassification of de novo LBCL were rendered using the revised $4^{\text {th }}$ edition 2016 World Health Organization Classification of Tumors Hematopoietic and Lymphoid Tissues. ${ }^{32}$ Clinical and outcome data for the LBCL cohort were acquired from the electronic medical record. Staging was classified according to revised International Pediatric Non-Hodgkin Lymphoma Staging System (IPNHLSS). ${ }^{33}$

\section{Immunohistochemistry}

Tissue microarrays were constructed for 68 cases. PD-L1 IHC was performed using the FDA-approved Dako PD-L1 IHC 28-8 pharmDx companion diagnostic assay per manufacturer established recommendations. PD-1 IHC staining (clone MRQ-22, Cell Marque) was performed at a 1:50 dilution on an automated Leica Bond III immunostainer. All PD-1/PD-L1 IHC results were independently reviewed and scored by two hematopathologists as previously described ${ }^{34}$ with a few modifications (Online Supplementary Table S1). Membranous PD-L1 staining of tumor cells and macrophages, and membranous PD-1 staining of tumor infiltrating lymphocytes (TIL) were scored for staining intensity: 0 (no staining), 1+ (weak or equivocal), 2+ (moderate), $3+$ (strong). Percent positivity was recorded 
as follows: the number of PD-L1-positive tumor cells out of total number of viable tumor cells (PD-L1-Tum); the area of PD-L1-positive macrophages out of total tumor area (PD-L1-Mac); number of PD-1-positive tumor infiltrating lymphocytes out of total number of tumor infiltrating lymphocytes (PD-1-TIL). The cut-offs for PD-1/PD-L1 positivity were $2+$ or $3+$ staining in $\geq 5 \%$ of tumor cells (PD-L1Tum) or $\geq 20 \%$ of macrophages (PD-L1-Mac) or TIL (PD-1-TIL). LBCL cases were classified based on cell of origin into germinal center B-cell (GCB) or non-GCB phenotypes according to the Hans IHC algorithm using CD10, BCL6, and MUM1 with a 30\% cut-off. ${ }^{35}$ Epstein-Barr virus (EBV) encoded RNA (EBER) in situ hybridization and LMP1 (latent membrane protein 1) staining were used to determine EBV status.

\section{Targeted next-generation sequencing and cytogenetics}

Targeted NGS mutation analysis using a custom-designed 152 gene (1.25 megabase [Mb]) panel for pediatric hematologic malignancies was performed on 13 cases of aggressive pediatric NHL (5 de novo LBCL not otherwise specified, 2 primary-immunodeficiency associated LBCL, 5 PTLDDLBCL, and $1 \mathrm{BL}$ ) as previously reported. ${ }^{36}$ Variants were classified as established or potential clinical significance (Tier I/II) or variants of uncertain significance (Tier III). ${ }^{37}$ Gains of 9p24.1 were determined from copy number analysis of the targeted sequencing data. 9p24.1 rearrangements were assessed for seven $L B C L$ cases using available cytogenetic data (Online Supplementary Table S2).

\section{Statistical analysis}

LBCL cases were classified as PD-L1 positive if PD-L1-Tum positive. Statistical analyses were performed using GraphPad Prism version 6.0.0. For the de novo LBCL and PTLDDLBCL cohorts, correlation between PD-L1-positivity and EBV status, cell of origin, stage, and nodal status was calculated using Fisher Exact test. Overall survival (OS) was defined as the time from diagnosis to death of any cause, and event-free survival (EFS) as the time to disease progression, relapse, or death of any cause, respectively. For survival analyses, Kaplan-Meier method with log-rank test was performed. Two-sided $P$-values $<0.05$ were considered statistically significant.

\section{Results}

\section{Diagnoses and clinicopathologic features}

A summary of the relevant clinicopathologic features, follow-up periods, and outcomes in the study cohort is provided in Table 1. Complete details are provided in the Online Supplementary Table S2. A total of 68 cases were studied; eight ALK+ALCL, $27 \mathrm{BL}$, and $33 \mathrm{LBCL}$ (consisting of de novo LBCL, $\mathrm{n}=22$; PTLD-DLBCL, $\mathrm{n}=11$ ). Patients ranged in age from 2.6 to 21.4 years (mean 10.6, median 10.8 years) with 45 males and 23 females. More than half of the cases were stage III/IV ( $n=41,61.2 \%)$ and extranodal involvement was documented in a majority of cases $(n=50,73.5 \%)$.

Of the patients with LBCL, the de novo LBCL cohort age ranged from 4.2 to 18.0 years (mean 11.6, median 11.3 years), with an equal number of males $(n=11)$ and females $(n=11)$. Diagnoses included DLBCL not otherwise specified (DLBCL-NOS, $n=14)$, PMBCL $(n=3)$, DLBCL with IGH $\alpha$-MYC rearrangement $(n=2)$, primary immunodeficiency-associated $L B C L$ (PIA-LBCL) arising in Ataxia-Telangiectasia $(n=2)$, and T-cell/histiocyte-rich LBCL (THRLBCL, $n=1)$; one PIA-LBCL was subclassified further as THRLBCL. Two de novo LBCL (9.1\%) were EBV+ and 15 (68.2\%) were classified as germinal center B-cell (GCB) phenotype including 10 DLBCL-NOS (71.4\%). De novo LBCL patients were treated according to various $\mathrm{COG}$ and POG protocols for pediatric B-cell NHL without Rituximab and three patients received bone marrow transplant (BMT) for relapsed/refractory disease (Online Supplementary Table S2). The follow-up period ranged from 4.2 to 253.9 months (mean 91.4, median 88.4 months).

Of the patients with LBCL, the PTLD-DLBCL cohort age ranged from 3.4 to 21.4 years (mean 12.9, median 15.9 years). Disease occurred after lung $(n=5)$, bone marrow $(n=2)$, renal $(n=2)$, liver $(n=1)$, or heart $(n=1)$ transplantation. Ten PTLD-DLBCL (90.9\%) were EBV+ and two (22.2\%) were classified as GCB. PTLD-DLBCL patients were treated according to various regimens that all included Rituximab (Online Supplementary Table S2). The follow up period ranged from 0.6 to 265.9 months (mean 61.1, median 50.0 months).

\section{PD-1/PD-L1 immunohistochemistry}

PD-L1 immunoreactivity in tumor cells (PD-L1-Tum) and macrophages (PD-L1-Mac), and PD-1 immunoreactivity in tumor infiltrating lymphocytes (PD-1-TIL) were assessed in the $A L K+A L C L, B L$, and $L B C L$ cohorts. A summary of the PD-1/PD-L1 IHC results is provided in Table 2. Representative IHC staining is shown in Figure 1.

\section{$P D-L 1-M a C$ and $P D-1-T I L$}

No ALK+ ALCL cases showed PD-L1 staining in macrophages. In the BL cohort, PD-L1-Mac positivity was observed in five cases (range, 30-60\%), but none of the PD-L1-Mac-positive BL demonstrated positive PD-L1-Tum staining. PD-L1-Mac positivity was seen in two DLBCLNOS (20\% each) and one PIA-LBCL that was classified as TCHRBCL (70\%) although neither PD-L1-Mac-positive DLBCL-NOS demonstrated positive PD-L1-Tum staining. PD-1-TIL positivity was observed in four cases (2 ALK+ ALCL, $1 \mathrm{BL}$, and 1 THRLBCL), and all cases occurred with concomitant PD-L1-Tum positivity. 
Table 1. Summary of study cohort $(\mathrm{N}=68)$.

\begin{tabular}{|c|c|c|c|c|c|}
\hline \multirow{2}{*}{ Diagnoses (count) } & \multirow{2}{*}{$\begin{array}{c}\text { Median age in years } \\
\text { (range) }\end{array}$} & \multirow{2}{*}{$\mathbf{M} \mid \mathbf{F}, \mathbf{N}$} & \multirow{2}{*}{ Stage III/IV, N } & \multicolumn{2}{|c|}{ Site of involvement, $\mathbf{N}(\%)$} \\
\hline & & & & Nodal & Extranodal \\
\hline ALK+ ALCL (8) & $12.7(6.7-15.8)$ & 612 & $5(62.5 \%)$ & $6(75.0 \%)$ & $2(25.0 \%)$ \\
\hline $\mathrm{BL}(27)$ & $8.4(2.6-18.5)$ & $24 \mid 3$ & $16(59.3 \%)$ & $2(7.4 \%)$ & $25(92.6 \%)$ \\
\hline $\begin{array}{l}\text { LBCL (33) } \\
\text { De novo (22) } \\
\text { PTLD-DLBCL (11) }\end{array}$ & $\begin{array}{l}11.7(3.4-21.4) \\
11.6(4.2-18.0) \\
12.9(3.4-21.4)\end{array}$ & $\begin{array}{c}15 \mid 18 \\
11 \mid 11 \\
4 \mid 7\end{array}$ & $\begin{array}{c}20(62.5 \%)^{*} \\
13(61.9 \%)^{*} \\
7(63.6 \%)\end{array}$ & $\begin{array}{c}10(30.3 \%) \\
6(27.3 \%) \\
4(36.4 \%)\end{array}$ & $\begin{array}{c}23(69.7 \%) \\
16(72.7 \%) \\
7(36.6 \%)\end{array}$ \\
\hline Totals (68) & $10.6(2.6-21.4)$ & $45 \mid 23$ & $41(61.2 \%)^{\star}$ & $18(26.5 \%)$ & $50(73.5 \%)$ \\
\hline \multicolumn{2}{|c|}{ LBCL classification } & Count (\%) & \multicolumn{2}{|c|}{ GCB, $\mathbf{N}(\%)$} & $E B V+, N(\%)$ \\
\hline \multicolumn{2}{|c|}{$\begin{array}{l}\text { De novo LBCL } \\
\text { DLBCL-NOS } \\
\text { PMBCL } \\
\text { THRLBCL } \\
\text { DLBCL with IGH } \alpha-M Y C \\
\text { PIA-LBCL^^}\end{array}$} & $\begin{array}{c}22(66.7 \%) \\
14 \\
3 \\
1 \\
2 \\
2\end{array}$ & $\begin{array}{l}15 / 2 \\
10 / 1\end{array}$ & & $\begin{array}{c}2 / 22(9.1 \%) \\
2 / 14(14.3 \%) \\
0 \\
0 \\
0 \\
0\end{array}$ \\
\hline \multicolumn{2}{|l|}{ PTLD-DLBCL } & $11(33.3 \%)$ & \multicolumn{2}{|c|}{$2 / 9(22.2 \%)^{\dagger}$} & $10 / 11(90.9 \%)^{\$}$ \\
\hline \multicolumn{2}{|l|}{ Totals } & $33(100.0 \%)$ & \multicolumn{2}{|c|}{$17 / 31(54.8 \%)^{\dagger}$} & $12 / 33(36.4 \%)^{\$}$ \\
\hline \multicolumn{2}{|l|}{ LBCL events } & All LBCL (33) & \multicolumn{2}{|c|}{ De novo (22) } & PTLD-DLBCL (11) \\
\hline \multicolumn{2}{|c|}{ Months follow-up (range) } & $80.7(0.6-265.9)$ & \multicolumn{2}{|c|}{$91.4(4.2-253.9)$} & $61.1(0.6-265.9)$ \\
\hline $\begin{array}{l}\text { Outcome, N (\% cohor } \\
\text { NED } \\
\text { REL, NED } \\
\text { REL, DOD } \\
\text { REF, DOD } \\
\text { DOD } \\
\text { DOC (transplant) }\end{array}$ & & $\begin{array}{c}21(63.6 \%) \\
4(12.1 \%) \\
2(6.1 \%) \\
1(3.0 \%) \\
4(12.1 \%) \\
1(3.0 \%)\end{array}$ & $\begin{array}{c}15 \\
3 \\
2 \\
1 \\
1\end{array}$ & & $\begin{array}{c}6(54.5 \%) \\
1(9.1 \%) \\
- \\
- \\
3(27.3 \%) \\
1(9.1 \%)\end{array}$ \\
\hline
\end{tabular}

*Staging was scored using International Pediatric Non-Hodgkin Lymphoma Staging System (IPNHLSS) criteria $^{32}$ and data were unavailable for 1 de novo PIA-LBCL. ^Both PIA-LBCL occurred in Ataxia-Telangiectasia patients. One PIA-LBCL was further subclassified as THRLBCL. †Cell of origin (GCB vs. non-GCB) classification for 2 cases of PTLD-DLBCL was unsuccessful due to insufficient tissue. ${ }^{\$}$ One EBV+ PTLD-DLBCL was positive for EBER (Epstein-Barr virus encoded RNA) only and negative for LMP-1 (latent membrane protein 1). ALK+ ALCL: ALK-positive anaplastic large cell lymphoma; BL: Burkitt lymphoma; DOC: died of complications; DOD: died of disease; DLBCL: diffuse large B-cell lymphoma; EBV+: Epstein-Barr virus positive; F: female; GCB: germinal center B-cell phenotype; LBCL: large B-cell lymphoma; M: male; NED: no evidence of disease; NOS: not otherwise specified; PIA: primary immunodeficiency-associated; PTLD: post-transplant lymphoproliferative disorder; PMBCL: primary mediastinal large B-cell lymphoma; REF: refractory; REL: relapsed; THRLBCL: T-cell/histocyte-rich large B-cell lymphoma.

Table 2. Summary of PD-1/PD-L1 immunohistochemistry results.

\begin{tabular}{|c|c|c|c|c|c|c|}
\hline Diagnoses (count) & $\begin{array}{c}\text { PD-L1-Tum } \geq 5 \% * \\
\text { N (\%) }\end{array}$ & Range \% & $\begin{array}{c}\text { PD-L1-Mac } \geq 20 \% \text { * } \\
\text { N (\%) }\end{array}$ & Range \% & $\begin{array}{c}\text { PD-1-TIL } \geq 20 \% * \\
\text { N (\%) }\end{array}$ & Range \% \\
\hline ALK+ ALCL (8) & $8(100.0 \%)$ & $30-100 \%$ & $00.0 \%$ & . & $225.0 \%$ & $20 \%$ \\
\hline $\mathrm{BL}(27)$ & $0(0.0 \%)$ & . & $518.5 \%$ & $30-60 \%$ & $00.0 \%$ & . \\
\hline LBCL (33) & 19 (57.6\%) & $30-100 \%$ & $39.1 \%$ & $20-70 \%$ & $13.0 \%$ & $20 \%$ \\
\hline $\begin{array}{l}\text { De novo LBCL (22) } \\
\text { DLBCL-NOS (14) } \\
\text { PMBCL (3) } \\
\text { THRLBCL (1) } \\
\text { DLBCL with IGH }- \text { MYC (2) } \\
\text { PIA-LBCL (2) }\end{array}$ & $\begin{array}{c}11(50.0 \%) \\
4 / 14(28.6 \%) \\
3 \\
1 \\
1 \\
2\end{array}$ & $\begin{array}{c}50-60 \% \\
50-90 \% \\
100 \% \\
30 \% \\
30-100 \%\end{array}$ & $\begin{array}{c}313.6 \% \\
2 \\
0 \\
0 \\
0 \\
1\end{array}$ & $\begin{array}{c}20 \% \\
\cdot \\
\cdot \\
\cdot \\
70 \%\end{array}$ & $\begin{array}{c}14.5 \% \\
0 \\
0 \\
1 \\
0 \\
0\end{array}$ & $20 \%$ \\
\hline PTLD-DLBCL (11) & $8(72.7 \%)$ & $40-100 \%$ & $00.0 \%$ & . & $00.0 \% \wedge$ & . \\
\hline
\end{tabular}

${ }^{*}$ Cases were considered PD-L1-Tum positive if PD-L1 membranous expression on tumor cells was $\geq 5 \%$, PD-L1-Mac positive if PD-L1 membranous expression on macrophages was $\geq 20 \%$, and PD-1-TIL positive if $\geq 20 \%$ tumor infiltrating lymphocytes showed membranous PD- 1 staining (Online Supplementary Table S1). ^Scoring PD-1 expression on tumor infiltrating lymphocytes (PD-1-TIL) was unsuccessful for one PTLD-DLBCL case due to insufficient tissue. ALK+ ALCL: ALK-positive anaplastic large cell lymphoma; BL: Burkitt lymphoma; DLBCL: diffuse large B-cell lymphoma; LBCL: large B-cell lymphoma; Mac: macrophages; NOS: not otherwise specified; PIA: primary immunodeficiency-associated; PDL1-Mac: PD-L1 expression on macrophages; PMBCL: primary mediastinal large B-cell lymphoma; PTLD: post-transplant lymphoproliferative disorder; THRLBCL: T-cell/histocyte-rich large B-cell lymphoma; TIL: tumor-infiltrating lymphocyte. 
PD-L1-TUM

As expected, all eight (100.0\%) of the ALK+ ALCL cases showed positive PD-L1-Tum staining (range, 30-100\%) and none of the $27 \mathrm{BL}$ cases (0.0\%). Overall, PD-L1-Tum positivity ranging from $30-100 \%$ was detected in $19 \mathrm{LBCL}$ (57.6\%) and 11 de novo LBCL (50.0\%) including four DLBCLNOS (28.6\%). Eight PTLD-DLBCL (72.7\%) showed PD-L1Tum-positive staining ranging from 40-100\%. In our cohort, none of the PD-L1-Tum-positive cases demonstrated less than $30 \%$ membranous positivity despite a $5 \%$ threshold for positivity. Three de novo LBCL cases showed a cytoplasmic PD-L1 staining pattern that was considered PD-L1-negative, and three LBCL showed essentially similar PD-L1 staining intensity and percent positivity on initial and relapsed/refractory lesions (data not shown).

\section{PD-L1 expression in pediatric large B-cell lymphomas}

LBCL cases were recorded as positive for PD-L1 expression only if PD-L1-Tum positivity was observed and subsequent statistical analyses focused on PD-L1-Tum staining status only (Table 3). PD-L1-Tum positivity significantly correlated with EBV+ LBCL $(P=0.033)$. There were no statistically significant differences between PD-
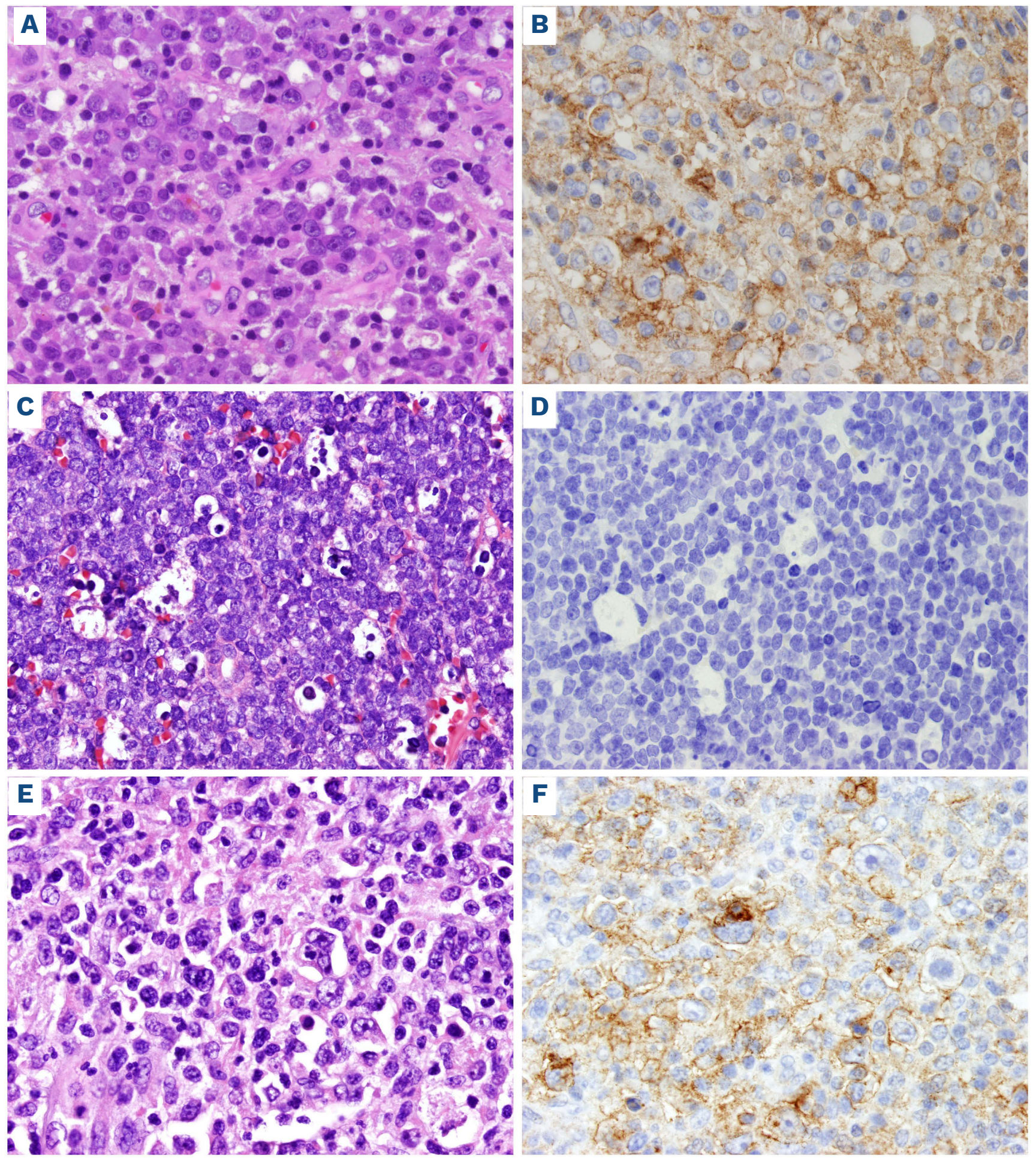

Figure 1. PD-L1 immunohistochemical stains in representative case of pediatric aggressive non-Hodgkin lymphomas. (A and $B$ ) ALK-positive anaplastic large cell lymphoma (ALK+ ALCL) showed tumor cells admixed with tumor microenvironment cells (lymphocytes and macrophages). Nearly all tumor cells show PD-L1 expression with moderate to strong staining intensity (2+ to 3+). ( $C$ and D) Burkitt lymphoma showed predominantly intermediate-sized lymphoma cells admixed with macrophages. PD-L1 showed no staining. (E and F) Epstein-Barr virus-postive diffuse large B-cell lymphoma not otherwise specified (EBV+ DLBCL-NOS) showed large lymphoma cells and PD-L1 overexpression in 50\% of tumor cells with moderate staining intensity (2+). (A to $F$ magnification 400x). 
L1-Tum staining and cell of origin, stage, or site of involvement in either the total cohort or within the de novo or PTLD-DLBCL subsets. The correlation between PD-L1Tum and OS and EFS was also examined (Figure 2). PDL1-Tum did not correlate with OS in the total LBCL cohort or within the de novo or PTLD-DLBCL subsets. Likewise, PD-L1-Tum was not significantly associated with EFS in the total LBCL cohort or in the PTLD-DLBCL subset. However, PD-L1 overexpression significantly correlated with lower EFS in the de novo LBCL subset $(P=0.017)$. Neither OS nor EFS could be calculated in the DLBCLNOS subset due to small sample size $(n=14)$ and number of events.

\section{Targeted next-generation sequencing}

In order to investigate the molecular mechanisms of PDL1 positivity in a subset of aggressive pediatric NHL, targeted NGS to assess for 9p24.1 copy number and TMB was performed on 12 LBCL (7 de novo LBCL [5 DLBCL-NOS, 2 PIA-LBCL] and 5 PTLD-DLBCL) and one BL. Cytogenetic data to assess for the presence of 9p24.1 gene rearrangements were reviewed for seven LBCL (5 de novo LBCL [4 DLBCL-NOS, 1 DLBCL with IGH@-MYC] and 2 PTLDDLBCL). Characteristic somatic ARID1A and TP53 and germline $A T M$ mutations were detected in the $B L^{38}$ and PIA-LBCL samples, respectively. One PTLD-DLBCL harbored an ARID1A mutation, another PTLD-DLBCL harbored

Table 3. Summary of PD-L1 statistical analyses for correlation study in large B-cell lymphoma cohort.

\begin{tabular}{|c|c|c|c|c|c|}
\hline LBCL cohort & Count & EBV+ / EBV- & GCB / non-GCB* & Stage I-II / III* & Nodal / Extranodal \\
\hline $\begin{array}{l}\text { De novo LBCL cohort } \\
\text { PD-L1-Tum POS } \\
\text { PD-L1-Tum NEG } \\
P \text {-value }\end{array}$ & $\begin{array}{l}11 \\
11 \\
-\end{array}$ & $\begin{array}{l}2 / 9 \\
0 / 11 \\
0.476\end{array}$ & $\begin{array}{l}7 / 4 \\
8 / 3 \\
1.000\end{array}$ & $\begin{array}{l}3 / 7 \\
5 / 6 \\
0.659\end{array}$ & $\begin{array}{l}2 / 9 \\
4 / 7 \\
0.635\end{array}$ \\
\hline $\begin{array}{l}\text { PTLD-DLBCL cohort } \\
\text { PD-L1-Tum POS } \\
\text { PD-L1-Tum NEG } \\
P \text {-value }\end{array}$ & $\begin{array}{l}8 \\
3 \\
-\end{array}$ & $\begin{array}{c}8 / 0 \\
2 / 1 \\
0.273\end{array}$ & $\begin{array}{l}1 / 6 \\
1 / 1 \\
0.417\end{array}$ & $\begin{array}{c}4 / 4 \\
0 / 3 \\
0.236\end{array}$ & $\begin{array}{c}4 / 4 \\
0 / 3 \\
0.236\end{array}$ \\
\hline $\begin{array}{l}\text { Total cohort } \\
\text { PD-L1-Tum POS } \\
\text { PD-L1-Tum NEG } \\
P \text {-value }\end{array}$ & $\begin{array}{c}19 \\
14 \\
-\end{array}$ & $\begin{array}{l}10 / 9 \\
2 / 12 \\
\mathbf{0 . 0 3 3}\end{array}$ & $\begin{array}{l}8 / 10 \\
9 / 4 \\
0.275\end{array}$ & $\begin{array}{l}7 / 11 \\
5 / 9 \\
1.000\end{array}$ & $\begin{array}{l}6 / 13 \\
4 / 10 \\
1.000\end{array}$ \\
\hline
\end{tabular}

*Cell of origin classification for 2 cases of PTLD-DLBCL was unsuccessful due to insufficient tissue. Staging was unavailable for 1 PIA-LBCL. LBCL: large B-cell lymphoma; EBV: Epstein-Barr virus; GCB: germinal center B-cell phenotype; PD-L1-Tum: PD-L1 expression on tumor cells; PTLD-DLBCL: diffuse large B-cell lymphoma arising as post-transplant lymphoproliferative disorder; POS: positive; NEG: negative.

Total LBCL $(n=33)$
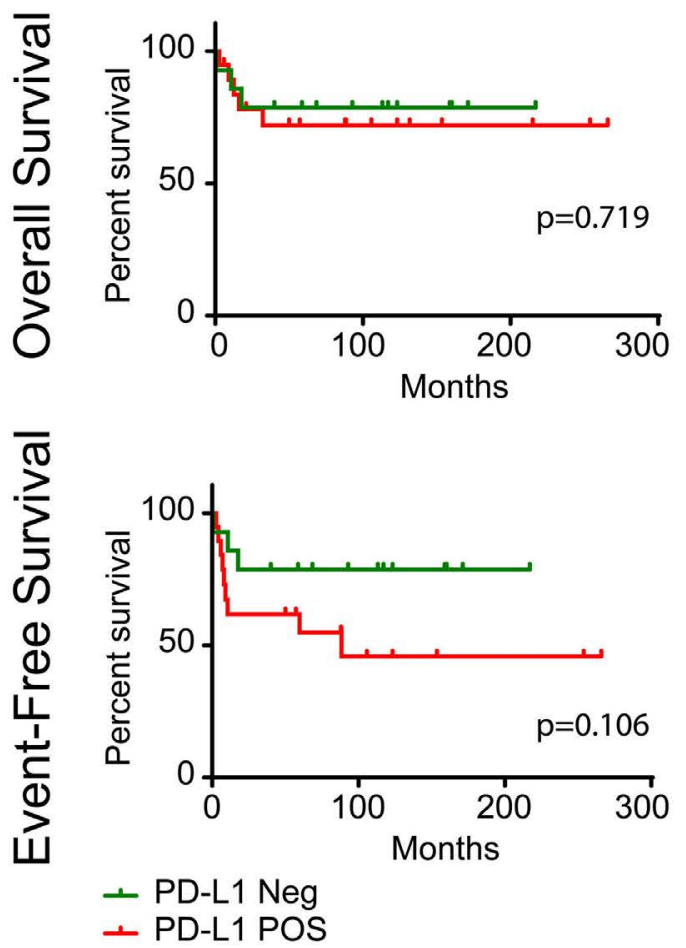

PTLD-LBCL $(n=11)$
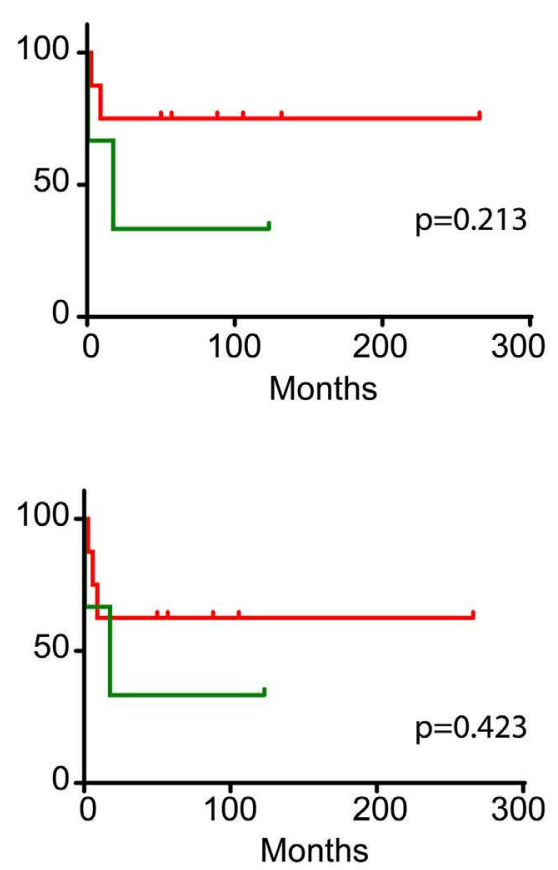

De Novo LBCL $(\mathrm{n}=22)$
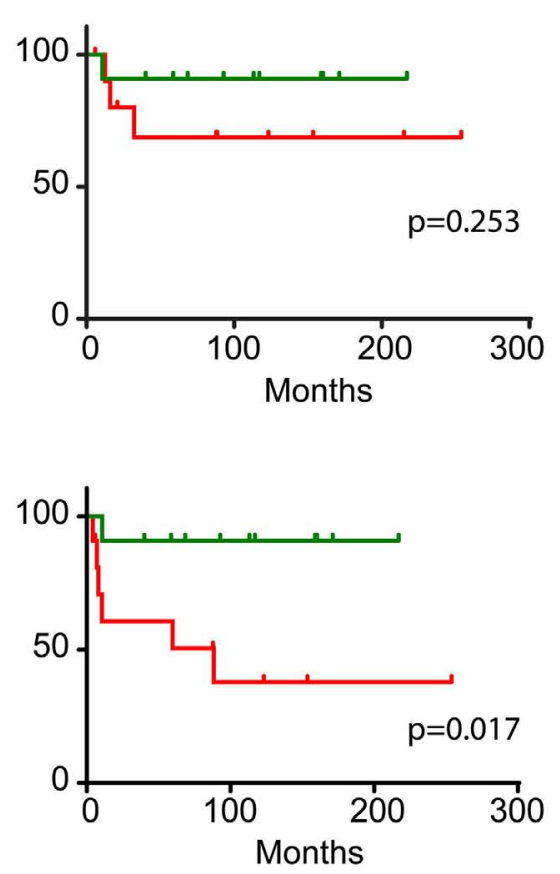

Figure 2. Kaplan-Meier survival curves. Overall survival (OS) and event-free survival (EFS) curves for PD-L1 staining in the total large B-cell lymphoma (LBCL), de novo LBCL, and diffuse LBCL arising as monomorphic post-transplant lymphoproliferative disorder (PTLD-DLBCL) cohorts. 
$D D X 3 X, I D 3$, and two TP53 co-mutations, and two DLBCLNOS cases showed mutations in CCND3 alone, and BCOR, CCND3, and U2AF1 co-mutations, respectively. The results are summarized in Table 4 and the Online Supplementary Table S3.

PD-L1-positive LBCL showed EBV positivity $(n=2)$, copy gains of the $9 p 24.1$ locus $(n=1)$, or both $(n=1)$. One PD-L1positive PIA-LBCL had no discernible mechanism for PDL1 overexpression and no 9p24.1 rearrangements were observed in any case. EBV positivity was observed in one PTLD-DLBCL and DLBCL-NOS without PD-L1 overexpression. Interestingly, one PTLD-DLBCL (LBCL-29) harbored an estimated TMB of 180 mutations per megabase $(\mathrm{Mb})$, which is considered an "ultra-hypermutated" tumor (>100 mutations/Mb). ${ }^{39}$ Pathogenic mutations in ASXL1, CDKN2A, CREBBP, FBXW7 $(\mathrm{n}=2), \mathrm{KMT} 2 \mathrm{C}, \mathrm{KMT2D}, \mathrm{KRAS}$, TP53 $(\mathrm{n}=2)$, and ZFHX3 as well as 104 coding or splicing variants of uncertain significance were detected in this tumor (Online Supplementary Table S3). Notably, this case did not show PD-L1-Tum positivity.

\section{Discussion}

Pembrolizumab is an FDA-approved PD-1 inhibitor for refractory childhood cHL. Likewise, the anti-PD-1 immune checkpoint inhibitor nivolumab has shown promising therapeutic efficacy in early phase clinical trials for pediatric lymphomas that overexpress PD-L1. ${ }^{23,24}$ Thus, defining PD-L1 staining patterns and cut-off positivity in aggressive pediatric NHLs using the 28-8 clone antibody, the complementary IHC diagnostic for nivolumab, is of potential clinical significance.

In this study, we defined positive PD-L1 staining as membranous staining of $5 \%$ of total viable tumor cells (PD-L1Tum). We observed positive PD-L1-Tum staining in all eight $A L K+A L C L$ and none of the $27 \mathrm{BL}$ consistent with previous reports. ${ }^{20,40}$ PD-L1-Tum positivity was observed in a ma-

Table 4. Summary of next-generation sequencing and 9p24.1 locus results.

\begin{tabular}{|c|c|c|c|c|c|c|c|c|}
\hline Sample & Diagnosis & PD-L1 & EBV+ & $\begin{array}{c}\text { GCB versus } \\
\text { non-GCB }\end{array}$ & $\begin{array}{c}9 \mathrm{p} 24.1 \\
\text { rearrangements }\end{array}$ & 9p24.1 gains & Tier I/II Mutations & VAF \\
\hline BL-21 & $\mathrm{BL}$ & Neg & Neg & . & $\mathrm{N} / \mathrm{A}$ & Not Detected & $\begin{array}{c}\text { ARID1A p.Y1679X } \\
\text { TP53 p.R175H }\end{array}$ & $\begin{array}{l}0.32 \\
0.75\end{array}$ \\
\hline LBCL-14* & PIA-LBCL^^ & Pos & Neg & non-GCB & $\mathrm{N} / \mathrm{A}$ & Detected & ATM p.S1924X $\$$ & 0.52 \\
\hline LBCL-9* & PIA-LBCL & Pos & $\mathrm{Neg}$ & non-GCB & Not Detected & Not Detected & $\begin{array}{l}\text { ATM p.W2638X } \$ \\
\text { ATM p.Q1970X }\end{array}$ & $\begin{array}{l}0.43 \\
0.52\end{array}$ \\
\hline LBCL-27 & PTLD-DLBCL & Pos & Pos & non-GCB & N/A & Detected & ARID1A p.Q172X & 0.40 \\
\hline LBCL-30 & PTLD-DLBCL & Pos & Pos & non-GCB & $\mathrm{N} / \mathrm{A}$ & Not Detected & None Detected & . \\
\hline LBCL-32 & PTLD-DLBCL & Pos & Pos & non-GCB & Not Detected & Not Detected & None Detected & \\
\hline LBCL-26 & PTLD-DLBCL & Neg & Pos & $\mathrm{N} / \mathrm{A}$ & $\mathrm{N} / \mathrm{A}$ & Not Detected & $\begin{array}{c}D D X 3 X \text { c. } 1171-2 \mathrm{~A}>\mathrm{G} \\
\text { ID3 p.L70Q } \\
\text { TP53 p.I50fs } \\
\text { TP53 p. } 1175 \mathrm{H}\end{array}$ & $\begin{array}{l}0.71 \\
0.43 \\
0.26 \\
0.35\end{array}$ \\
\hline LBCL-17* & DLBCL-NOS & Neg & $\mathrm{Neg}$ & non-GCB & Not Detected & Not Detected & None Detected & . \\
\hline LBCL-16* & DLBCL-NOS & Neg & $\mathrm{Neg}$ & GCB & Not Detected & Not Detected & CCND3 p.R271fs & 0.17 \\
\hline LBCL-12* & DLBCL-NOS & Neg & $\mathrm{Neg}$ & GCB & $\mathrm{N} / \mathrm{A}$ & Not Detected & None Detected & . \\
\hline LBCL-13* & DLBCL-NOS & Neg & Neg & GCB & N/A & Not Detected & $\begin{array}{c}\text { BCOR p.N1459S } \\
\text { CCND3 p.R271fs } \\
\text { U2AF1 p.S34F }\end{array}$ & $\begin{array}{l}0.89 \\
0.41 \\
0.45\end{array}$ \\
\hline LBCL-3* & DLBCL-NOS & Neg & Pos & GCB & $N / A$ & Not Detected & None Detected & \\
\hline $\mathrm{LBCL}^{-29^{\dagger}}$ & PTLD-DLBCL & Neg & Neg & GCB & Not Detected & Not Detected & 11 total, high $\mathrm{TMB}^{\dagger}$ & 0.43 \\
\hline
\end{tabular}

*Denotes de novo LBCL. ^Further subclassified as T-cell/histiocyte-rich large B-cell lymphoma: †Ultra-hypermutated tumor with 180 mutations per megabase (Mb). A complete list of mutations is provided in the Online Supplementary Table S3. ${ }^{\$} \mathrm{Known}$ germline. BL: Burkitt lymphoma; DLBCL: diffuse large B-cell lymphoma; GCB: germinal center B-cell phenotype; LBCL: large B-cell lymphoma; N/A: not available; Neg: negative; Pos: positive; PTLD: post-transplant lymphoproliferative disorder; TMB: tumor mutation burden; VAF: variant allele fraction. 
jority of LBCL including $50.0 \%$ of de novo LBCL. The percentage of PD-L1 positive DLBCL-NOS cases in our pediatric cohort (28.6\%) was slightly higher than two recent adult studies in which positive PD-L1 staining was reported in approximately $10-15 \%$ of DLBCL-NOS cases. ${ }^{29,31}$ The frequency of PD-L1 positive pediatric PTLD-DLBCL cases $(72.7 \%)$ was similar to other pediatric and adult PTLD-DLBCL cohorts that reported approximately $60-80 \%$ positivity. ${ }^{13,34,41}$

Despite defining $5 \%$ as the threshold for PD-L1-positivity, all PD-L1-positive cases in our cohort showed at least $30 \%$ positive membranous staining. Kiyasu et al. ${ }^{29} \mathrm{em}-$ ployed a similar cut-off in adult DLBCL and reported PDL1 positivity ranges from $30-100 \%$ while observing a marked decrease in staining below the $30 \%$ threshold. Additionally, we detected minimal PD-L1-Mac staining or PD-1-TIL staining consistent with other pediatric studies that reported low levels of PD-1-positivity. ${ }^{34}$ Together, the data support a potential role for PD-L1 testing in pediatric aggressive NHL, including ALK+ ALCL, de novo LBCL, and PTLD-DLCBL. Furthermore, our data suggest that a positivity threshold of $30 \%$ is suitable when assessing pediatric aggressive NHL for PD-L1 staining of tumor cells.

We correlated LBCL PD-L1-Tum staining with multiple clinicopathologic features although the small number of cases precluded comprehensive statistical analyses. There was no statistically significant correlation between stage, nodal status, cell of origin, or OS in the total LBCL cohort, or when separated into de novo LBCL and PTLDDLBCL. PD-L1-Tum staining correlated with EBV positivity in our study for the entire $L B C L$ cohort, but not with PTLD-DLBCL cohort alone. Although PTLD-DLBCL comprised a large portion of the EBV+ $L B C L$ samples in our cohort, the grouping of EBV+ de novo LBCL and PTLD$\angle B C L$ is not necessarily representative of clinical practice. Nonetheless, correlation between EBV positivity and PDL1 overexpression in pediatric LBCL was previously reported; Nicolae et $a .^{42}$ reported a strong correlation in patients younger than 45 years of age between EBV+ and PD-L1 expression in LBCL, particularly in THRBCL and nonGCB DLBCL, which was recapitulated here. Furthermore, $\angle B C L$ classified as intrinsic (oncogenic) induction of TME correlated with EBV positivity suggesting that EBV is a potent regulator of PD-L1 in pediatric LBCL.

In two previous adult $L B C L$ studies in which a higher threshold for tumor cell positivity was utilized (30\%), PDL1 positivity correlated with inferior OS. ${ }^{29,31}$ Using a de facto cut-off of $30 \%$ in our study, PD-L1 staining correlated with reduced EFS in de novo LBCL, although no statistical significance in OS was observed. However, studies investigating the correlation between PD-L1 positivity and survival in adult DLBCL are confounded by multiple variables such as the antibody clone employed, IHC scoring methodology adopted, and/or non-uniform positivity thresholds. ${ }^{29-31,43}$ We acknowledge that conclusions regarding $O S$ and EFS are limited due to the retrospective nature of our study, the small numbers of LBCL subtypes, and non-uniform treatment regimens, particularly pediatric intensive treatment regimens without rituximab for de novo LBCL treatment. Also, the grouping of diagnostic entities that have distinct clinicopathologic and therapeutic considerations (e.g., PMBCL) restricts the generalization of the statistical findings. Nonetheless, the negative correlation between PD-L1 and EFS in pediatric de novo LBCL was observed, and additional studies are warranted to establish PD-L1-Tum as a negative prognostic marker in clinical pediatric practice. CD274 gene overexpression and increased PD-L1 protein translation can occur through a variety of mechanisms such as increased CD274 transcription via promoter activation or CD274 copy gains, amplifications, and gene rearrangements. ${ }^{17,18,20,21}$ In our study, PD-L1 staining was observed most frequently in cases with EBV positivity and 9p24.1 copy gains. However, in two EBV+ cases, one de novo LBCL and one PTLD-DLBCL, no PD-L1 staining was observed. This is not unexpected, as EBV-associated CD274 gene regulation requires complex interplay between transcriptional elements, microRNA, and post-transcriptional modifications. ${ }^{44,45}$ Also, in one PD-L1-positive PIALBCL case no 9p24.1 copy gains or rearrangements were observed. Thus, it is possible that this case represented a false-negative 9p24.1 result; comprehensive molecular assessments of CD274 3'-UTR structural variations and gene rearrangements ${ }^{46}$ not detectable by karyotype analysis were not performed.

Interestingly, we encountered an EBV-negative PTLDDLBCL with no PD-L1 positivity with an estimated TMB of 180 mutations/Mb consistent with an "ultra-hypermutated" tumor (>100 mutations/Mb). The high TMB was not attributable to somatic hypermutation; all mutations were detected in coding exons and not in immunoglobulin heavy chain and light chain variable region genes. ${ }^{47}$ The accuracy of TMB assessment is proportional to the number of bases sequenced so smaller targeted panels can lead to imprecise TMB scores. However, recent studies suggest that highly accurate TMB assessments can be obtained with targeted panels larger than 1.0 Mb. ${ }^{48,49}$ In this study, we utilized a targeted sequencing panel that captures and sequences approximately 1.25 million individual bases (1.25 Mb) so we conclude that our TMB estimates are reasonably accurate. Hypermutation is rare in pediatric tumors; however when detected, it often occurs in the setting of congenital mismatch repair deficiency (CMMRD) syndromes and/or acquisition of somatic POLE/POLD1 mutations ${ }^{39}$ but genetic testing for CMMRD or POLE/POLD1 somatic mutation testing was not performed in this case. Despite the lack of PD-L1 positivity in this case, emerging data suggest that either PD-L1 
staining or high TMB should be considered positive biomarkers for response to immune checkpoint inhibitor therapy. ${ }^{50}$ Collectively, our data indicate that EBV and 9p24.1 copy gains are key regulators of PD-L1 overexpression in a subset of pediatric LBCL, particularly in PTLD-DLBCL, which is consistent with other studies in adult patients. ${ }^{40}$ Assessment of TMB may identify some patients that may benefit from immune checkpoint inhibitor therapy in the absence of PD-L1-Tum staining. The genomic landscape of pediatric LBCL has not been extensively studied, but in adults, mutations in DLCBL tend to cluster in pathways that regulate cell signaling and growth, B-cell development, and nucleic acid transcription and translation. ${ }^{51}$ Our sequencing analysis revealed similar somatic pathogenic mutations regulators of the cell cycle, transcription, and DNA repair (CCND3, $D D X 3 X, I D 3, T P 53)$, and mRNA splicing (U2AF1), but also identified mutations in epigenetic modifiers (ARID1A, $B C O R)$. There did not appear to be significant differences in the types of mutations encountered in GCB versus nonGCB cases or differences in mutational profiles between de novo LBCL or PTLD-DLBCL. However, only 152 genes were sequenced in our study and the panel did not include several genes that were recently reported to cluster in discreet LBCL subtypes (e.g., B2M, CD79B, CIITA, GNAI2, and SGK1). ${ }^{52,53}$ Thus, the limited gene content and small number of cases hinders a definitive differential assessment of mutational signatures.
In conclusion, as one of the largest pediatric series to date, we showed that $30 \%$ cut-off for membranous PDL1 staining in the tumor cells (PD-L1-Tum) is an appropriate threshold to assess for PD-L1 positivity and correlated with EBV positivity in LCBL and lower EFS in de novo LBCL. Also, EBV and copy gains of 9p24.1 appear to regulate PD-L1 overexpression in a subset of cases and rare cases of pediatric NHL may demonstrate high TMB highlighting a potential new biomarker in this disease. Additional studies exploring the therapeutic and prognostic significance, molecular mechanisms, and host environments that affect PD-1/PD-L1 staining in aggressive pediatric $\mathrm{NHL}$ are warranted.

\section{Disclosures}

No conflicts of interest to disclose.

\section{Contributions}

KEF and CVC designed the study, wrote, edited the manuscript, and supervised the project. $L S F, A M C, A M M$, and BYM performed experiments and collected and analyzed data. JLC, ANM, DLT, and KYK assisted in data collection and provided clinical expertise.

\section{Data sharing statement}

Data sharing is subject to Institutional policies governing clinical research data.

\section{References}

1. Weber J. Immune checkpoint proteins: a new therapeutic paradigm for cancer - preclinical background: CTLA-4 and PD-1 blockade. Semin Oncol. 2010;37(5):430-439.

2. Xia Y, Medeiros LJ, Young KH. Immune checkpoint blockade: releasing the brake towards hematological malignancies. Blood Rev. 2016;30(3):189-200.

3. Xie W, Medeiros LJ, Li S, Yin CC, Khoury JD, XU J. PD-1/PD-L1 pathway and its blockade in patients with classic Hodgkin lymphoma and non-Hodgkin large-cell lymphomas. Curr Hematol Malig Rep. 2020;15(4):372-381.

4. Jin HT, Ahmed R, Okazaki T. Role of PD-1 in regulating T-cell immunity. Curr Top Microbiol Immunol. 2011;350:17-37.

5. Keir ME, Butte MJ, Freeman GJ, Sharpe AH. PD-1 and its ligands in tolerance and immunity. Annu Rev Immunol. 2008;26:677-704.

6. Pardoll DM. The blockade of immune checkpoints in cancer immunotherapy. Nat Rev Cancer. 2012;12(4):252-264.

7. Topalian SL, Drake CG, Pardoll DM. Immune checkpoint blockade: a common denominator approach to cancer therapy. Cancer Cell. 2015;27(4):450-461.

8. Bryan LJ, Gordon LI. Blocking tumor escape in hematologic malignancies: the anti-PD-1 strategy. Blood Rev.

2015;29(1):25-32.

9. Afreen S, Dermime S. The immunoinhibitory B7-H1 molecule as a potential target in cancer: killing many birds with one stone. Hematol Oncol Stem Cell Ther. 2014;7(1):1-17.

10. Sznol M, Chen L. Antagonist antibodies to PD-1 and B7-H1 (PDL1) in the treatment of advanced human cancer. Clin Cancer Res. 2013;19(5):1021-1034.

11. Xia Y, Jeffrey Medeiros L, Young KH. Signaling pathway and dysregulation of PD1 and its ligands in lymphoid malignancies. Biochim Biophys Acta. 2016;1865(1):58-71.

12. Shen J, Li S, Medeiros LJ, et al. PD-L1 expression is associated with ALK positivity and STAT3 activation, but not outcome in patients with systemic anaplastic large cell lymphoma. Mod Pathol. 2020;33(3):324-333.

13. Kinch A, Sundström C, Baecklund E, Backlin C, Molin D, Enblad G. Expression of PD-1, PD-L1, and PD-L2 in posttransplant lymphoproliferative disorder after solid organ transplantation. Leuk Lymphoma. 2019;60(2):376-384.

14. Chan TA, Yarchoan M, Jaffee E, et al. Development of tumor mutation burden as an immunotherapy biomarker: utility for the oncology clinic. Ann Oncol. 2019;30(1):44-56.

15. Rizvi NA, Hellmann MD, Snyder A, et al. Cancer immunology. Mutational landscape determines sensitivity to PD-1 blockade in non-small cell lung cancer. Science. 2015;348(6230):124-128.

16. Georgiou K, Chen L, Berglund M, et al. Genetic basis of PD-L1 overexpression in diffuse large B-cell lymphomas. Blood. 
2016;127(24):3026-3034.

17. Green MR, Monti S, Rodig SJ, et al. Integrative analysis reveals selective 9p24.1 amplification, increased PD-1 ligand expression, and further induction via JAK2 in nodular sclerosing Hodgkin lymphoma and primary mediastinal large Bcell lymphoma. Blood. 2010;116(17):3268-3277.

18. Roemer MG, Advani RH, Ligon AH, et al. PD-L1 and PD-L2 genetic alterations define classical Hodgkin lymphoma and predict outcome. J Clin Oncol. 2016;34(23):2690-2697.

19. Kataoka K, Shiraishi Y, Takeda Y, et al. Aberrant PD-L1 expression through 3 '-UTR disruption in multiple cancers. Nature. 2016;534(7607):402-406.

20. Chen BJ, Chapuy B, Ouyang J, et al. PD-L1 expression is characteristic of a subset of aggressive B-cell lymphomas and virus-associated malignancies. Clin Cancer Res. 2013;19(13):3462-3473.

21. Green MR, Rodig S, Juszczynski P, et al. Constitutive AP-1 activity and EBV infection induce PD-L1 in Hodgkin lymphomas and posttransplant lymphoproliferative disorders: implications for targeted therapy. Clin Cancer Res. 2012;18(6):1611-1618.

22. Atsaves V, Tsesmetzis N, Chioureas D, et al. PD-L1 is commonly expressed and transcriptionally regulated by STAT3 and MYC in ALK-negative anaplastic large-cell lymphoma. Leukemia. 2017;31(7):1633-1637.

23. Davis KL, Fox E, Merchant MS, et al. Nivolumab in children and young adults with relapsed or refractory solid tumours or lymphoma (ADVL1412): a multicentre, open-label, single-arm, phase 1-2 trial. Lancet Oncol. 2020;21(4):541-550.

24. Geoerger B, Kang HJ, Yalon-Oren M, et al. Pembrolizumab in paediatric patients with advanced melanoma or a PD-L1positive, advanced, relapsed, or refractory solid tumour or lymphoma (KEYNOTE-051): interim analysis of an open-label, single-arm, phase 1-2 trial. Lancet Oncol. 2020;21(1):121-133.

25. Hamanishi J, Mandai M, Matsumura N, Abiko K, Baba T, Konishi I. PD-1/PD-L1 blockade in cancer treatment: perspectives and issues. Int J Clin Oncol. 2016;21(3):462-473.

26. Davis KL, Agarwal AM, Verma AR. Checkpoint inhibition in pediatric hematologic malignancies. Pediatr Hematol Oncol. 2017;34(6-7):379-394.

27. Luchtel RA, Dasari S, Oishi N, et al. Molecular profiling reveals immunogenic cues in anaplastic large cell lymphomas with DUSP22 rearrangements. Blood. 2018;132(13):1386-1398.

28. Teng MW, Ngiow SF, Ribas A, Smyth MJ. Classifying cancers based on T-cell infiltration and PD-L1. Cancer Res. 2015;75(11):2139-2145.

29. Kiyasu J, Miyoshi H, Hirata A, et al. Expression of programmed cell death ligand 1 is associated with poor overall survival in patients with diffuse large B-cell lymphoma. Blood. 2015;126(19):2193-2201.

30. Kwon D, Kim S, Kim PJ, et al. Clinicopathological analysis of programmed cell death 1 and programmed cell death ligand 1 expression in the tumour microenvironments of diffuse large $B$ cell lymphomas. Histopathology. 2016;68(7):1079-1089.

31. Xing W, Dresser K, Zhang R, et al. PD-L1 expression in EBVnegative diffuse large $B$-cell lymphoma: clinicopathologic features and prognostic implications. Oncotarget. 2016;7(37):59976-59986.

32. Swerdlow SH, Campo E, Pileri SA, et al. The 2016 revision of the World Health Organization classification of lymphoid neoplasms. Blood. 2016;127(20):2375-2390.

33. Angelo Rosolen A, Perkins SK, Pinkerton CR, et al. Revised International Pediatric Non-Hodgkin Lymphoma Staging System. J Clin Oncol. 2015:33(18):2112-2118.

34. Schiefer Al, Salzer E, Füreder A, et al. PD-L1 and PD1 expression in post-transplantation lymphoproliferative disease (PTLD) of childhood and adolescence: an inter- and intra-individual descriptive study covering the whole spectrum of PTLD categories. Cancer Med. 2019;8(10):4656-4668.

35. Hans CP, Weisenburger DD, Greiner TC, et al. Confirmation of the molecular classification of diffuse large B-cell lymphoma by immunohistochemistry using a tissue microarray. Blood. 2004;103(1):275-282.

36. Zhou T, Bloomquist MS, Ferguson LS, et al. Pediatric myeloid sarcoma: a single institution clinicopathologic and molecular analysis. Pediatr Hematol Oncol. 2020;37(1):76-89.

37. Li MM, Datto M, Duncavage EJ, et al. Standards and guidelines for the interpretation and reporting of sequence variants in cancer: a joint consensus recommendation of the Association for Molecular Pathology, American Society of Clinical Oncology, and College of American Pathologists. J Mol Diagn. 2017;19(1):4-23.

38. Giulino-Roth L, Wang K, MacDonald TY, et al. Targeted genomic sequencing of pediatric Burkitt lymphoma identifies recurrent alterations in antiapoptotic and chromatin-remodeling genes. Blood. 2012;120(26):5181-5184.

39. Shlien A, Campbell BB, de Borja R, et al. Combined hereditary and somatic mutations of replication error repair genes result in rapid onset of ultra-hypermutated cancers. Nat Genet. 2015;47(3):257-262.

40. Marzec M, Zhang Q, Goradia A, et al. Oncogenic kinase NPM/ALK induces through STAT3 expression of immunosuppressive protein CD274 (PD-L1, B7-H1). Proc Natl Acad Sci U S A. 2008;105(52):20852-20857.

41. Veloza L, Teixido C, Castrejon N, et al. Clinicopathological evaluation of the programmed cell death 1 (PD1)/programmed cell death-ligand 1 (PD-L1) axis in post-transplant lymphoproliferative disorders: association with Epstein-Barr virus, PD-L1 copy number alterations, and outcome. Histopathology. 2019;75(6):799-812.

42. Nicolae A, Pittaluga S, Abdullah S, et al. EBV-positive large Bcell lymphomas in young patients: a nodal lymphoma with evidence for a tolerogenic immune environment. Blood. 2015;126(7):863-872.

43. McCord R, Bolen CR, Koeppen H, et al. PD-L1 and tumorassociated macrophages in de novo DLBCL. Blood Adv. 2019;3(4):531-540.

44. Garcia-Lacarte M, Grijalba SC, Melchor J, Arnaiz-Leché A, Roa S. The PD-1/PD-L1 checkpoint in normal germinal centers and diffuse large B-cell lymphomas. Cancers (Basel). 2021;13(18):4683.

45. Li X, Zhang W. Expression of PD-L1 in EBV-associated malignancies. Int Immunopharmacol. 2021;95:107553.

46. Chong LC, Twa DD, Mottok A, et al. Comprehensive characterization of programmed death ligand structural rearrangements in B-cell non-Hodgkin lymphomas. Blood. 2016;128(9):1206-1213.

47. Xu-Monette ZY, Li J, Xia Y, et al. Immunoglobulin somatic hypermutation has clinical impact in DLBCL and potential implications for immune checkpoint blockade and neoantigenbased immunotherapies. J Immunother Cancer. 2019;7(1):272.

48. Buchhalter I, Rempel E, Endris V, et al. Size matters: dissecting key parameters for panel-based tumor mutational burden analysis. Int J Cancer. 2019;144(4):848-858.

49. Endris V, Buchhalter I, Allgäuer M, et al. Measurement of tumor mutational burden (TMB) in routine molecular diagnostics: in silico and real-life analysis of three larger gene panels. Int $\mathrm{J}$ Cancer. 2019;144(9):2303-2312.

50. Yarchoan M, Albacker LA, Hopkins AC, et al. PD-L1 expression 
and tumor mutational burden are independent biomarkers in most cancers. JCI Insight. 2019;4(6):e126908.

51. Reddy A, Zhang J, Davis NS, et al. Genetic and functional drivers of diffuse large B cell lymphoma. Cell. 2017;171(2):481494.

52. Kotlov N, Bagaev A, Revuelta MV, et al. Clinical and biological subtypes of B-cell lymphoma revealed by microenvironmental signatures. Cancer Discov. 2021;11(6):1468-1489.

53. Wright GW, Huang DW, Phelan JD, et al. A probabilistic classification tool for genetic subtypes of diffuse large B cell lymphoma with therapeutic implications. Cancer Cell. 2020;37(4):551-568. 